\title{
On the measure of nonclassicality of field states
}

\author{
J.M.C. Malbouisson ${ }^{(1)}$ and B. Baseia ${ }^{(2)}$ \\ (1) Instituto de Física, Universidade Federal da Bahia, 40210-340, Salvador, BA, Brazil \\ (2) Instituto de Física, Universidade Federal da Goiás, 74001-970, Goiânia, GO, Brazil \\ E-mail: jmalboui@ufba.br, basilio@fis.ufg.br
}

PACS Ref: 03.65.Bz, 42.50.Dv, 42.65.Ky

\begin{abstract}
The degree of nonclassicality of states of a field mode is analysed considering both phase-space and distance-type measures of nonclassicality. By working out some general examples, it is shown explicitly that the phase-space measure is rather sensitive to superposition of states, with finite superpositions possessing maximum nonclassical depth (the highest degree of nonclassicality) irrespective to the nature of the component states. Mixed states are also discussed and examples with nonclassical depth varying between the minimum and the maximum allowed values are exhibited. For pure Gaussian states, it is demonstrated that distance-type measures based on the HilbertSchmidt metric are equivalent to the phase-space measure. Analyzing some examples, it is shown that distance-type measures are efficient to quantify the degree of nonclassicality of non-Gaussian pure states.
\end{abstract}




\section{Introduction}

Since the first observations of nonclassical properties of electromagnetic field states, like subPoissonian statistics [1] and squeezing [2], theoretical interest has been growing in the search of a way to measure the degree of nonclassicality of a given state. Generally, one can classify a state as nonclassical if its Glauber-Sudarsham $P$-function [3] has negative values or if it is more singular than a delta function. However, the nonclassical properties do not occur simultaneously for all the states; for example, squeezed-vacuum states are super-Poissonian while the odd coherent states are sub-Poissonian but do not present quadrature squeezing, and so the question how much nonclassical a given state is seems to be appropriated.

Several proposals have been made to quantify how much nonclassical a state can be. Restricted to the photon statistics, Mandel [4] introduced a parameter $\left(q=\left\langle\hat{n}^{2}\right\rangle /\langle\hat{n}\rangle-\langle\hat{n}\rangle-1\right)$ quantifying the departure from the Poissonian behavior of a coherent (quasi-classical) state; such a measure, however, does not contemplate other nonclassical properties of field states. Hillery [5], defined the nonclassical distance of a state as the trace norm of the difference between the density operator of the state and that of the nearest classical state. In practical calculations, however, the determination of the nearest classical state is rather difficult. Other more operational measures of nonclassicality, following this trend, have been introduced by Dodonov et al. [6], using the Hilbert-Schmidt distance between density operators and, more recently, by Marian et al. [7], employing the Bures-Uhlmann definition of distance between states. Distinctly, inspired in the Cahill-Glauber representation [8], Lee [9] introduced the $R$-function as a (real) $\tau$-parametrized 
Gaussian convolution of the $P$-function and defined the nonclassical depth of a state as the minimum value of $\tau$ for which the $R$-function becomes a nonnegative definite function, thus acceptable as a classical distribution function. A similar phase-space measure of nonclassicality was also considered by Lütkenhaus and Barnett [10]. Nonclassical measure of nonclassical properties [11] and an observable criterion to distinguish nonclassical states [12] have also been discussed recently.

This article focuses initially on the phase-space measure of nonclassicality of one-mode field states as firstly introduced by Lee [9]. After a brief review of the phase-space criterium, by analyzing some general cases, it is explicitly shown that this measure is rather sensitive to superposition of states, with finite superpositions of states having the highest degree of nonclassicality (maximum nonclassical depth) irrespective to the nature of the component states. Next, the nonclassical depth of mixed states is considered and examples with nonclassical depth varying between the minimum and the maximum permitted values are presented. The paper proceeds with the analysis

of distance-type measures. It is demonstrated that distance-type measures based on the HilbertSchmidt metric are equivalent to the phase-space measure for pure Gaussian states (squeezed states). Then, it is shown that they provide a way of quantifying the degree of nonclassicality for non-Gaussian ones. Finally, some conclusions are drawn.

\section{Phase-space measure of nonclassicality}

By noticing that Gaussian convolutions of the $P$-function, as the Wigner $(W)$ and the Husimi $(Q)$ functions, are regular functions of a complex variable $z$ and inspired in the Cahill-Glauber repre- 
sentation [8], Lee [9] introduced a continuous family of representations of a field state depending on one parameter $(\tau \geq 0)$ as the set of Gaussian convolutions of the $P$-function

$$
R(z ; \tau)=\frac{1}{\pi \tau} \int d^{2} w \exp \left(-\frac{1}{\tau}|z-w|^{2}\right) P(w)
$$

$\tau=\frac{1}{2}$ and 1 correspond to the $W$ - and the $Q$-functions respectively, while one recovers the $P$-function in the limit $\tau \rightarrow 0$. The fact that the $W$-function is regular but usually possesses negative values and that the $Q$-function is always a non-negative definite function leads to the definition of the nonclassical depth of a given state as the minimum value of $\tau\left(\tau_{\mathrm{m}}\right)$ for which the corresponding $R$-function is non-negative in the whole $z$-plane, thus becoming acceptable as a classical distribution function. From this definition, it follows that $0 \leq \tau_{\mathrm{m}} \leq 1$, examples of limiting cases being coherent and thermal states, with $\tau_{\mathrm{m}}=0$, and number states which have maximum nonclassical depth $\left(\tau_{\mathrm{m}}=1\right)$. As non extreme examples, squeezed states possess $\tau_{\mathrm{m}}$ ranging from 0 to $\frac{1}{2}$ as the squeezing parameter $\left(e^{|\zeta|}\right)$ varies from 1 to $\infty$. Not only this criterium is well-defined mathematically but also it indicates how to calculate the degree of nonclassicality (the nonclassical depth) of a given state of the field.

If one works in the number basis, with matrix elements of the density operator of a given state denoted by $\rho(n, m)=\langle n|\hat{\rho}| m\rangle$, the $R$-functions are given by 13

$$
R(z ; \tau)=\frac{1}{\pi \tau} e^{-|z|^{2} / \tau} \sum_{n, m=0}^{\infty} \rho(n, m) e^{i(m-n) \theta} A_{n, m}
$$

where $z=|z| e^{i \theta}$

$$
A_{n, m}=\left(\frac{m !}{n !}\right)^{1 / 2} \frac{(\tau-1)^{m}}{\tau^{n}}|z|^{n-m} L_{m}^{n-m}\left(\frac{|z|^{2}}{\tau(\tau-1)}\right), n \leq m
$$


$L_{m}^{n-m}$ denoting the generalized Laguerre polynomial, and with $A_{n>m}=A_{m, n}$. This expression is very convenient to calculate the $R$-functions of finite superpositions of number states. On the other hand, in the coherent basis, the $R$-functions can be written as [9]

$$
\begin{aligned}
R(z ; \tau)=\frac{1}{\pi(1-\tau)} & \exp \left(\frac{|z|^{2}}{1-\tau}\right) \int d^{2} \beta\langle-\beta|\hat{\rho}| \beta\rangle \\
& \times \exp \left(-\frac{1}{1-\tau}\left[(2 \tau-1)|\beta|^{2}+\left(z^{*} \beta-z \beta^{*}\right)\right]\right) .
\end{aligned}
$$

For a general finite superposition of coherent states,

$$
|\psi\rangle=\sum_{i=1}^{N} c_{i}\left|\alpha_{i}\right\rangle,
$$

performing some Gaussian integrals, the Eq. (四) reduces to

$$
\begin{gathered}
R(z ; \tau)=\frac{1}{\pi \tau} \sum_{i, j=1}^{N} c_{i} c_{j}^{*} \exp \left(-\frac{1}{2}\left[\left|\alpha_{i}\right|^{2}+\left|\alpha_{j}\right|^{2}-2 \alpha_{i} \alpha_{j}^{*}\right]\right) \\
\times \exp \left(-\frac{1}{\tau}\left(z-\alpha_{i}\right)\left(z^{*}-\alpha_{j}^{*}\right)\right) .
\end{gathered}
$$

This formula is useful to calculate the nonclassical depth of superpositions of coherent states.

\section{$3 \quad$ Nonclassical depth of superposed states}

Since the $R$-functions are certainly regular functions of $z$ for $\tau \geq 1 / 2$, a general procedure to find the nonclassical depth of a state ( $\tau_{\mathrm{m}}$ as defined before) consists in determining the minimum value of the function $R(z ; \tau)$ for a sequence of values of $\tau$ and then search for the smallest value of $\tau \in(0,1]$ for which the minimum value of $R\left(z ; \tau_{\mathrm{m}}\right)$ vanishes. In many cases, where one suspects 
that $\tau_{\mathrm{m}}=1$ (maximum nonclassical depth), one may investigate how the value of $R(z ; \tau)$ at a chosen point behaves as $\tau$ is increased inside the interval $(0,1]$. Next, the nonclassical depth of some examples of superposed states of the electromagnetic field are calculated.

\subsection{General superposition of the vacuum and the $n$-photons state}

As a first example, consider superpositions of the vacuum $|0\rangle$, a state with minimum degree of nonclassicality $\left(\tau_{\mathrm{m}}=0\right)$, with the number state $|n\rangle$, which has maximum nonclassical depth $\left(\tau_{\mathrm{m}}\right.$ $=1$ ). Take the set of normalized states

$$
|\psi(\xi ; \phi)\rangle_{0, n}=\sqrt{\xi}|0\rangle+\sqrt{1-\xi} e^{i \phi}|n\rangle
$$

where $0 \leq \xi \leq 1$ and $\phi$ is an arbitrary relative phase. These states continuously interpolate from $|0\rangle$ to $|n\rangle$, so one wonders what happens to the nonclassical depth of such states, as the parameter $\xi$ varies from 0 to 1 . From Eqs. (2) and (3), it follows that the $R$-function corresponding to the state (7) is given by

$$
\begin{aligned}
R_{|\psi\rangle_{0, n}}(z ; \tau)=\frac{1}{\pi \tau} e^{-|z|^{2} / \tau} & {\left[\xi L_{0}\left(\frac{|z|^{2}}{\tau(\tau-1)}\right)+(1-\xi)\left(\frac{\tau-1}{\tau}\right)^{n} L_{n}\left(\frac{|z|^{2}}{\tau(\tau-1)}\right)\right.} \\
& \left.+2 \sqrt{\xi(1-\xi)}\left(\frac{|z|}{\tau}\right)^{n} L_{0}^{n}\left(\frac{|z|^{2}}{\tau(\tau-1)}\right) \cos (n \theta-\phi)\right],
\end{aligned}
$$

and one can analyze its minimum value for various cases.

For the simplest one, $n=1$, using that $L_{0}(x)=1, L_{1}(x)=1-x$ and $L_{0}^{1}(x)=1$, Eq. (8) reduces to 


$$
R_{|\psi\rangle_{0,1}}(z ; \tau)=\frac{1}{\pi \tau} e^{-|z|^{2} / \tau}\left[\frac{(1-\xi)}{\tau^{2}}|z|^{2}+\frac{2 \sqrt{\xi(1-\xi)}}{\tau} \cos (\theta-\phi)|z|+\frac{\xi+\tau-1}{\tau}\right] .
$$

One sees that the sign of the minimum value of $R_{|\psi\rangle_{0,1}}$ is dictated by the term inside the square bracket above. If one chooses the worse circumstance as far as the positiveness of the function $R_{|\psi\rangle_{0,1}}$ is concerned, $\cos (\theta-\phi)=-1$ (corresponding to look at the axis in the $z$-plane defined by $\theta=\phi+\pi)$, one sees that the minimum of the quadratic function of $|z|$ in the square bracket above is attained at $|z|=\tau \sqrt{\xi /(1-\xi)}$ and has value $(1-\xi)(\tau-1) / \tau$, which is negative for all $0<\tau<1$ for any $0 \leq \xi<1$, being zero only when $\tau=1$. Therefore, the states $|\psi(\xi ; \phi)\rangle_{0,1}$ possess $\tau_{\mathrm{m}}=1$ for all $0 \leq \xi<1$ and such states are as nonclassical as possible. It is impressive the fact that, no matter how close the value of $\xi$ is to 1 , independently of the relative phase $\phi$, the state $|\psi(\xi ; \phi)\rangle_{0,1}$ is as much nonclassical as it is a number state, in particular the state $|1\rangle$. In contrast, the Mandel parameter for the superposition (7) is given by $q_{|\psi\rangle_{0, n}}=-1+n \xi$ and so, for $n=1$, it varies continuously from -1 to 0 as $\xi$ goes from 0 to 1 . Analyzing the minimum of the function (8), one can verify that $\tau_{\mathrm{m}}=1$ is maintained for the cases $n>1$. One is then led to speculate that the phase-space measure of nonclassicality is rather sensitive to superposition of states and that maximum nonclassical depth is more a rule than an exception. To check this assertion, the superposition of two quasi-classical states are investigated next. 


\subsection{General superposition of opposite coherent states}

Consider a general superposition of opposite coherent states ( $\alpha$ real for simplicity) given by

$$
|\psi(\xi, \phi)\rangle=\mathcal{N}\left(\sqrt{\xi}|-\alpha\rangle+\sqrt{1-\xi} e^{i \phi}|\alpha\rangle\right)
$$

for which $\mathcal{N}=\left(1+2 \sqrt{\xi(1-\xi)} \cos \phi e^{-2 \alpha^{2}}\right)^{-1 / 2}$; such state interpolates between the coherent states $|\alpha\rangle$ and $|-\alpha\rangle$ as $\xi$ varies from 0 to 1 . For $|\alpha| \gg 1,\langle-\alpha \mid \alpha\rangle=\exp \left(-2 \alpha^{2}\right) \approx 0, \mathcal{N} \cong 1$ and such states correspond to mesoscopic superpositions of quasi-classical states known as Schrödingercat states of the field. In the particular case where $\xi=1 / 2$ and $\phi=0(\phi=\pi)$, this superposition is called even (odd) coherent state and possesses well-defined parity. Since both component states have minimal nonclassical depth, $\tau_{\mathrm{m}}=0$, it is worth looking at the nonclassical depth of the superposition. Using expression (6), one has

$$
R_{|\psi\rangle}(z ; \tau)=\frac{1}{\pi \tau}\left(e^{\alpha^{2}}+2 \sqrt{\xi(1-\xi)} \cos \phi e^{-\alpha^{2}}\right)^{-1} \exp \left(-\frac{1}{\tau}\left(x^{2}+y^{2}\right)\right) F(x, y ; \tau ; \xi ; \phi)
$$

where $z=x+i y$ and

$$
\begin{aligned}
F(x, y ; \tau ; \xi ; \phi)=\exp ( & \left.-\frac{(1-\tau)}{\tau} \alpha^{2}\right)\left[\exp \left(\frac{2 \alpha x}{\tau}\right)-2 \xi \sinh \left(\frac{2 \alpha x}{\tau}\right)\right] \\
& +2 \sqrt{\xi(1-\xi)} \exp \left(\frac{(1-\tau)}{\tau} \alpha^{2}\right) \cos \left(\frac{2 \alpha y}{\tau}-\phi\right) .
\end{aligned}
$$

The function $F$, which determines the sign of the R-function (11), possesses minima located at points $x=\tau[\ln \xi-\ln (1-\xi)] / 4 \alpha$ and $y=[(2 n+1) \pi+\phi] \tau / 2 \alpha$, where $n$ is an integer. The minimum values attained are all identical and given by $F_{\min }=-4 \sqrt{\xi(1-\xi)} \sinh \left[\alpha^{2}(1-\tau) / \tau\right]$, 
which is negative for all $0<\tau<1$, for $0<\xi<1$ and independently of $\phi$, no matter the value of $\alpha$. Therefore, one concludes that superpositions of opposite coherent states possess maximum nonclassical depth $\left(\tau_{\mathrm{m}}=1\right)$, irrespective of the value of $\alpha$, notwithstanding the fact that components of these superpositions are as classical as possible, having $\tau_{\mathrm{m}}=0$. It is amazing that a superposition of two quasi-classical states (with $\tau_{\mathrm{m}}=0$ ), no matter how further apart they are, is as nonclassical as possible, with the same nonclassical depth $\left(\tau_{\mathrm{m}}=1\right)$ as that for a number state. It seems though that just the fact of considering a superposition of states, a concept lying at the heart of Quantum Mechanics, is enough to lead to maximum degree of nonclassicality in the sense of the phase-space measure.

Many other examples can be treated, corroborating with the point discussed above: superpositions of coherent and number states, of squeezed and number states, of coherent and squeezed states and so on. In all such cases, one finds maximum nonclassical depth. Also, higher-generation Schrö dinger-cat states of a field mode [14] possess maximum degree of nonclassicality in the context of the phase-space measure. As another important example, it has been shown that all Pegg-Barnett phase-states [15] have the greatest nonclassical depth $\left(\tau_{\mathrm{m}}=1\right)$, irrespective to the dimension of the truncated Hilbert space they are defined [16, 17]. Thus the phase-space criterium of nonclassicality for pure states of the field looks like an "all-or-nothing" measure, with $\tau_{\mathrm{m}}$ being either 0 or 1 , with the exception of squeezed states. Also, it is clear that no direct relation between the phase-space measure of nonclassicality and the presence of some specified nonclassical property can exist; for example, a state with $\tau_{\mathrm{m}}=1$ can be super-Poissonian (as phase states) or 
can present no quadrature squeezing, as happens with number states.

It is worth comment on the equivalence between the phase-space measure of nonclassicality as stated by Lee [9] and by Lütkenhaus and Barnett [10]. In the later, maximum nonclassicality is signalized by the existence of zeros in the $Q$-function while Lee's criterium for maximum nonclassical depth can be stated by saying that the $R$-functions for all $\tau<1$ possess negative regions. The fact that $R(z ; \tau)$, for all $z$, are continuous functions of the parameter $\tau$ ensures the mentioned equivalence. For the simple examples analyzed here, one can easily follow the disappearance of the negative regions of the $R$-functions as $\tau$ approaches 1 . Actually, by analyzing the zeros of the Husimi $Q$-function, it can be proved that all quantum states which are not Gaussian have maximum degree of nonclassicality in this phase-space measure [10. The insensitivity of the phase-space measure to distinguish between pure, non-Gaussian, states raises the question whether other type of measure may be more efficient to do so. As it will be shown in Section 5 ,

measures based on notions of distance between states are able to quantify in a broader sense the nonclassicality of pure states. But, before presenting this analysis, the phase-space measure of nonclassicality of mixed states is considered.

\section{Nonclassical depth of mixed states}

Now, turn the attention to mixed states. Clearly, since the phase-space representations are linear in the density operator, if one considers a mixture of states with all parcels possessing $\tau_{\mathrm{m}}=0$, the nonclassical depth is maintained minimal. In the same way, mixtures of states having maximum 
nonclassical depth possess $\tau_{\mathrm{m}}=1$. On the other hand, Lee [13 showed that any state (pure or not) for which $\rho(0,0)=\langle 0|\hat{\rho}| 0\rangle=0$ possesses maximum nonclassical depth. An explicit example is the mixed state described in Ref. [18], which interpolates between a number and a chaotic (thermal) state. So, only mixtures containing a non-null vacuum part need to be analyzed and, for simplicity, take general mixtures of $|0\rangle$ and a number state $|n\rangle$, specified by

$$
\hat{\rho}=\xi|0\rangle\langle 0|+(1-\xi)| n\rangle\langle n| ;
$$

such mixed states also interpolates between $|0\rangle$ and $|n\rangle$, but in a distinct fashion from that of the state ( 7). The $R$-functions, in this case, are given by

$$
R_{\hat{\rho}}(z ; \tau ; \xi)=\frac{1}{\pi \tau} e^{-|z|^{2} / \tau}\left[\xi+(1-\xi)\left(\frac{1-\tau}{\tau}\right)^{n}(-1)^{n} L_{n}\left(\frac{|z|^{2}}{\tau(1-\tau)}\right)\right]
$$

and the investigation of the minimum value of the function within the square bracket leads to the nonclassical depth.

In the extremes, $\xi=0$ and $\xi=1$, the nonclassical depth are 1 and 0 , respectively, while for $0<\xi<1$, it is found that $\tau_{\mathrm{m}}^{(n)}(\xi)$ varies continuously between 1 and 0 . For small $n$, $\tau_{\mathrm{m}}^{(n)}$ can be calculated analytically and one finds, for example, $\tau_{\mathrm{m}}^{(1)}(\xi)=1-\xi$ and $\tau_{\mathrm{m}}^{(2)}(\xi)=$ $(\sqrt{\xi(1-\xi)}-1+\xi) /(2 \xi-1)$. Fig. 1 illustrates the behavior of $\tau_{\mathrm{m}}^{(n)}(\xi)$ for some values of $n$. One sees that, as $n$ increases, the nonclassical depth grows for $\frac{1}{2}<\xi<1$, showing that the nonclassicality of the number state dominates the scenario when $n$ is large even when the weight of the vacuum part in the mixture is bigger than that of $|n\rangle$. There is a competition between the positiveness of the $R$-function of the vacuum (for all $0 \leq \tau \leq 1$ ) and the existence of negative 
regions in the $R$-function of the number state $|n\rangle$ when $0<\tau<1$ but, in a sense, the number state is favored.

As far as the nonclassical properties of the states (13) are concerned, it is interesting to notice that the Mandel factor is given by $q(\xi, n)=\xi n-1$ (the same expression as that for the superposition (7), as expected) and one sees that super-Poissonian statistics occurs for all $n \geq 2$ if $\frac{1}{n}<\xi<1$; for $\xi>\frac{1}{2}$, the super-Poissonian character grows as $n$ increases, even though the states become more nonclassical within the phase-space measure. Furthermore, these states do not present quadrature squeezing in any circumstance. This shows that no direct relation between the nonclassical depth and these nonclassical properties exists. Also, there is no relation between the nonclassical depth and the degree of impurity $D=\operatorname{Tr}\left(\hat{\rho}-\hat{\rho}^{2}\right)$, measuring the departure from the idempotent property; states (13) have $D=2 \xi(1-\xi)$ independently of $n$, while $\tau_{\mathrm{m}}^{(n)}(\xi)$ grows as $n$ increases.

Other situations can be analyzed (as mixtures of coherent and number states, of squeezed and number states, and so on) suggesting that a mixture of two states has nonclassical depth varying continuously in the interval defined by the nonclassical depth of the added states. But there are exceptions, for example a mixture of a state with $\tau_{\mathrm{m}}=0$ (e.g. a coherent state) and a squeezed state. This is because the $R$-functions of a squeezed state $|\zeta\rangle$ becomes singular when $\tau<\tau_{\mathrm{m}}^{(\zeta)}$ 的 and the positiveness of the $R$-functions of states with $\tau_{\mathrm{m}}=0$ can not compensate this singular behavior. In such cases, the nonclassical depth of the mixture is equal to $\tau_{\mathrm{m}}^{(\zeta)}$. For the same reason, a mixture of two squeezed states has nonclassical depth equal to the largest value of $\tau_{\mathrm{m}}$ of 
its parts.

\section{Distance-type measures of nonclassicality}

There are other measures of nonclassicality defined as functions of the distance between the state and a conventionalized set of all classical states of the field mode. Such kind of measure was firstly introduced by Hillery [5], with the degree of nonclassicality being given by the trace norm of the difference between density operators of the state and of its nearest classical state. The determination of the nearest classical state, however, is rather difficult to be implemented. A more operational way to introduce distance-type measures of nonclassicality consists in to elect a subset of the space of the density operators as the set of most classical states, choosing a welldefined distance function in it, and to define the degree of nonclassicality as the minimum value of a monotonically increasing function of the distance between the state and a representative (arbitrary) nonclassical state.

Some definitions of distance between states have been used in Quantum Optics, important examples being the Hilbert-Schmidt distance,

$$
d^{\mathrm{HS}}(\hat{\rho}, \hat{\sigma})=\sqrt{\operatorname{Tr}\left\{(\hat{\rho}-\hat{\sigma})^{2}\right\}},
$$

and the Bures-Uhlmann distance,

$$
d^{\mathrm{BU}}(\hat{\rho}, \hat{\sigma})=\sqrt{2-2 \operatorname{Tr}\left\{(\sqrt{\hat{\rho}} \hat{\sigma} \sqrt{\hat{\rho}})^{1 / 2}\right\}} .
$$


These metrics have been employed to define distance-type measures of nonclassicality by Dodonov et al. [6] and by Marian et al. [7], respectively. For pure states, $|\psi\rangle$ and $|\varphi\rangle$, these distances are expressed as functions of the quantum-mechanical transition probability between the states, $|\langle\psi \mid \varphi\rangle|^{2}$; the Hilbert-Schmidt distance reduces to

$$
d^{\mathrm{HS}}(|\psi\rangle,|\varphi\rangle)=\sqrt{2-2|\langle\psi \mid \varphi\rangle|^{2}}
$$

while the Bures-Uhlmann distance becomes

$$
d^{\mathrm{BU}}(|\psi\rangle,|\varphi\rangle)=\sqrt{2-2|\langle\psi \mid \varphi\rangle|}
$$

In dealing with pure states, the natural choice as the set of most nonclassical states consists of the set of all coherent states, $\{|\beta\rangle\}$. Since the minimum of any monotonically increasing function of the distance between the state and the set of most nonclassical ones can be used as a measure of nonclassicality, here,

$$
d_{\mathrm{m}}=\min _{\{|\beta\rangle\}}\left[1-|\langle\psi \mid \beta\rangle|^{2}\right]=1-\pi \max _{\{\beta \in \mathbf{C}\}} Q_{|\psi\rangle}(\beta)
$$

where $Q_{|\psi\rangle}(\beta)$ is the Husimi $Q$-function corresponding to $|\psi\rangle$, will be used to quantify the degree of nonclassicality of the pure state $|\psi\rangle$. This definition slightly differs from the measures of nonclassicality introduced in Refs. [6] and [7]; it just avoids unnecessary square roots and it is normalized such that $0 \leq d_{\mathrm{m}}<1$, making easy the comparison with the nonclassical depth $\tau_{\mathrm{m}}$. Naturally, the minimal value $\left(d_{\mathrm{m}}=0\right)$ occurs for coherent states. For a number state $|n\rangle$ one has $d_{\mathrm{m}}^{(n)}=1-n^{n} e^{-n} / n$ !, so that all number states have different degrees of nonclassicality, the upper 
limit value been reached only when $n \rightarrow \infty$; this feature does not happens within the context of the phase-space measure. Notice that, while the nonclassical depth $\left(\tau_{\mathrm{m}}\right)$ is obtained from the minimum of the $R$-function, the most nonclassical states having zeros in the corresponding $Q$-functions, $d_{\mathrm{m}}$ is determined from the maximum value of the Husimi $Q$-function.

These measures of nonclassicality, however, are equivalent to each other if one restricts the analysis to the set of Gaussian pure states. Indeed, for a Stoler squeezed state

$$
|\alpha, \zeta\rangle=\hat{D}(\alpha) \hat{S}(\zeta)|0\rangle
$$

with $\hat{D}(\alpha)=\exp \left(\alpha \hat{a}^{\dagger}-\alpha^{*} \hat{a}\right)$ and $\hat{S}(\zeta)=\exp \left(\frac{1}{2} \zeta \hat{a}^{\dagger 2}-\frac{1}{2} \zeta^{*} \hat{a}^{2}\right)$, the $Q$-function is given by (setting $\left.\zeta=r e^{i \theta}\right)$

$$
\begin{aligned}
Q_{|\alpha, \zeta\rangle}(\beta)= & \frac{\operatorname{sech} r}{\pi} \exp \left[-(1-\tanh r \cos \theta) x^{2}\right. \\
& \left.-(1+\tanh r \cos \theta) y^{2}+2 \tanh r \sin \theta x y\right],
\end{aligned}
$$

where $x=\operatorname{Re}(\beta-\alpha)$ and $y=\operatorname{Im}(\beta-\alpha)$. The maximum value of this function is equal to $\pi^{-1} \operatorname{sech} r$ and is reached when $\beta=\alpha$. Therefore, for a squeezed state,

$$
d_{\mathrm{m}}^{(\zeta)}=1-\operatorname{sech} r
$$

In terms of the nonclassical depth for a squeezed state 91,

$$
\tau_{\mathrm{m}}^{(\zeta)}=\frac{\tanh r}{1+\tanh r}
$$

this quantity can be written as

$$
d_{\mathrm{m}}^{(\zeta)}=1-\sqrt{\frac{1-2 \tau_{\mathrm{m}}^{(\zeta)}}{\left(1-\tau_{\mathrm{m}}^{(\zeta)}\right)^{2}}},
$$


that is, $d_{\mathrm{m}}^{(\zeta)}$ is a bijective function of $\tau_{\mathrm{m}}^{(\zeta)}$, which proves the equivalence between these two measures of nonclassicality for pure Gaussian states; $\tau_{\mathrm{m}}^{(\zeta)}$ goes from 0 to $1 / 2$ and $d_{\mathrm{m}}^{(\zeta)}$ varies from 0 to 1 as $r$ raises from 0 to $\infty$. This equivalence has also been demonstrated for a Bures-Uhlmann distancetype measure [7]; this indicates that phase-space and distance-type measures of nonclassicality are equivalent for the set Gaussian pure states. Actually, all measures based on distances that, for pure states, depend only on $|\langle\psi \mid \varphi\rangle|$, with coherent states elected as the most nonclassical ones, are equivalent to the phase-space measure as far squeezed states are concerned.

On the other hand, for non-Gaussian pure states, like the superpositions of states analyzed before with the phase-space measure, such an equivalence does not exist, an aspect already observed for number states. In fact, for the general superposition of the vacuum and the one-photon state

$$
|\psi(\xi ; \phi)\rangle_{0,1}=\sqrt{\xi}|0\rangle+\sqrt{1-\xi} e^{i \phi}|1\rangle
$$

the $Q$-function is given by (taking $\beta=b e^{i \theta}$ )

$$
Q_{|\psi\rangle_{0,1}}(\beta)=\frac{1}{\pi} e^{-b^{2}}\left[\xi+(1-\xi) b^{2}+2 \sqrt{\xi(1-\xi)} b \cos (\theta-\phi)\right]
$$

and the degree of nonclassicality (19) is found to be

$$
d_{\mathrm{m}}^{(0,1)}(\xi)=1-\exp \left[-\frac{2-\xi-\sqrt{\xi(4-3 \xi)}}{2(1-\xi)}\right]\left[1+\frac{1}{2} \sqrt{\xi(4-3 \xi)}-\frac{1}{2} \xi\right]
$$

One sees that $d_{\mathrm{m}}^{(0,1)}(\xi)$ decreases monotonically from $d_{\mathrm{m}}^{(1)}=1-e^{-1}$ (corresponding to the number state $|1\rangle)$ to $d_{\mathrm{m}}^{(0)}=0$ (the degree of nonclassicality of the vacuum) as $\xi$ varies from 0 to 1 , a behavior rather distinct from the phase space-measure which gives $\tau_{\mathrm{m}}^{(0,1)}=1$ for all $0 \leq \xi<1$. 
This example shows clearly that distance-type measures are efficient to quantify the degree of nonclassicality of non-Gaussian pure states, at least for measures based on the Hilbert-Schmidt distance, in contrast with the phase-space measure which assigns maximum nonclassical depth to all of them.

As another example, consider the even $(+)$ and the $(-)$ coherent states (Schrödinger-cat states with well-defined parities), with $\alpha \in \mathbf{R}^{+}$for simplicity,

$$
|\Psi\rangle_{ \pm}=\frac{1}{\sqrt{2\left[1 \pm \exp \left(-2 \alpha^{2}\right)\right]}}(|-\alpha\rangle+|\alpha\rangle) .
$$

Their $Q$-functions, obtained from Eqs. (11) and (12) by making $\tau=1, \xi=1 / 2$ and $\phi_{+}=0$ (or $\left.\phi_{-}=\pi\right)$, are given by

$$
Q_{ \pm}(x, y)=\frac{\exp \left[-\left(x^{2}+y^{2}\right)\right]}{\pi\left[\exp \left(\alpha^{2}\right) \pm \exp \left(-\alpha^{2}\right)\right]}[\cosh (2 \alpha x) \pm \cos (2 \alpha y)]
$$

For even states, maximum values of the functions $Q_{+}$, which are certainly attained at points along the $y=0$ line, can not be analytically calculated for arbitrary $\alpha$, but the degree of nonclassicality can be easily evaluated by numerical means. For $0 \leq \alpha \leq 1$, maxima occur at $x=0$ and one finds exactly

$$
d_{\mathrm{m}}^{(+)}(\alpha)=1-\operatorname{sech}\left(\alpha^{2}\right)
$$

When $\alpha$ is increased above 1, two maxima of the function $Q_{+}$(for each $\alpha$ ) exist at values of $x$ which approaches $-\alpha$ and $\alpha$; for $\alpha=2.7$, maxima occurs at $|x|=2.699997$ in a six decimal-places precision. Thus, asymptotically (for large $\alpha$ )

$$
d_{\mathrm{m}}^{(+)}(\alpha) \simeq \frac{1}{2}\left[1-\exp \left(-2 \alpha^{2}\right)\right]
$$


and one sees that the degree of nonclassicality, relative to the measure ( 19), of even coherent states grows continuously from 0 to $1 / 2$ as $\alpha$ varies from 0 to $\infty$, while $\tau_{\mathrm{m}}^{(+)}=1$ for all $\alpha>0$. The increasing of $d_{\mathrm{m}}^{(+)}(\alpha)$, meaning that the even coherent state becomes more nonclassical as $\alpha$ augments, is in agreement with the fact that the decoherence time diminishes as the separation (in the phase space) of the components of a superposition of coherent states is enlarged [19. Similarly, investigating the maxima of $Q_{-}(x, y)$ as $\alpha$ is changed, one finds that the degree of nonclassicality of odd coherent states, $d_{\mathrm{m}}^{(-)}(\alpha)$, varies continuously from the value $d_{\mathrm{m}}^{(1)}=1-e^{-1}$ (corresponding to the number state $|1\rangle$ for which $|\Psi\rangle_{\text {_ }}$ tends when $\left.\alpha \rightarrow 0\right)$ to $1 / 2$ as $\alpha$ is increased from 0 to $\infty$.

The analysis of the degree of nonclassicality of mixed states using distance-type measures is more involved since, in this case, one has to use the expressions (15) or (16), which are much less operational than (17) and (18), and to extend the set of the most nonclassical states [6, [7]. This issue and the discussion of the relation between the degree of nonclassicality and the occurrence of nonclassical properties are left for future work.

\section{Conclusions}

The phase-space measure of nonclassicality of states of a field mode has been discussed for both superpositions and mixtures of pure states. General superpositions of the vacuum and number states and of opposite coherent states were considered to show explicitly that pure non-Gaussian states possess maximum nonclassical depth, while mixtures of representative pure states have (in general) nonclassical depth interpolating between the values corresponding to their added 
parts. Distance-type measures were also analyzed. It was demonstrated that all measures of nonclassicality defined as the minimum value of any monotonically increasing function of a distance between the state and the set of all coherent states, which can be expressed sole in terms of the quantum-mechanical transition probability between the states, are equivalent to the phasespace measure when one deals with pure-Gaussian (squeezed) states, the degree of nonclassicality being a bijective function of the nonclassical depth. For non-Gaussian pure states, examples were examined showing that distance-type measures are more appropriated to quantify the degree of nonclassicality in this case. The more involving problem of quantifying the nonclassicality of mixed states within the viewpoint of distance-type measures will be discussed elsewhere.

\section{Acknowledgments}

This work was partially supported by CNPq, Brazil.

\section{References}

[1] Short, R. and Mandel, L., Phys. Rev. Lett. 51, 384 (1983).

[2] Stoler, D., Phys. Rev. D 1, 3217 (1970); Yuen, H. P., Phys. Rev. A 13, 2226 (1976); Walls, D. F., Nature 306, 141 (1983).

[3] Glauber, R. J., Phys. Rev. Lett. 10, 84 (1963); Sudarshan, E. C. G., Phys. Rev. Lett. 10, $277(1963)$. 
[4] Mandel, L., Opt. Lett. 4, 205 (1979).

[5] Hillery, M., Phys. Rev. A 35, 725 (1987); Phys. Rev. A 39, 2994 (1989).

[6] Dodonov, V. V., Man'ko, O. V., Man'ko, V. I. and Wünsche, A., Physica Scripta 59, 81 (1999); J. Mod. Opt. 47, 633 (2000).

[7] Marian, P., Marian, T. A. and Scutaru, H., Phys. Rev. Lett. 88, 153601 (2002).

[8] Cahill, K. E. and Glauber, R. J., Phys. Rev. 177, 1857 (1969); Phys. Rev. 177, 1882 (1969).

[9] Lee, C. T., Phys. Rev. A 44, R2775 (1991).

[10] Lütkenhaus, N. and Barnett, S. M., Phys. Rev. A 51, 3340 (1995).

[11] Kim, K., Phys. Rev. A 59, 1566 (1999).

[12] Vogel, W., Phys. Rev. Lett. 84, 1849 (2000).

[13] Lee, C. T., Phys. Rev. A 52, 3374 (1995).

[14] Malbouisson, J. M. C. and Baseia, B., J. Mod. Opt. 46, 2015 (1999).

[15] Pegg, D. T. and Barnett, S. M., Europhys. Lett. 6, 483 (1988); J. Mod. Opt. 36, 7 (1988).

[16] Marchiolli, M. A., Bagnato, V. S., Guimarães, Y. and Baseia, B., Phys. Lett. A 279, 294 (2001).

[17] Malbouisson, J. M. C., Phys. Lett. A 286, 405 (2001). 
[18] Baseia, B., Duarte, S. B. and Malbouisson, J. M. C., J. Opt. B: Quantum Semiclass. Opt. 3, $152(2001)$.

[19] Brune, M. et al., Phys. Rev. Lett. 77, 4887 (1996).

\section{Figure caption}

Fig. 1. Nonclassical depth $\left(\tau_{\mathrm{m}}\right)$ versus the interpolating parameter $(\xi)$ for mixtures of the vacuum and a $n$-photons state, Eq. ( 13). Dotted, dashed, dot-dashed and full lines correspond to $n=1,2,3$ and 4 , respectively. 


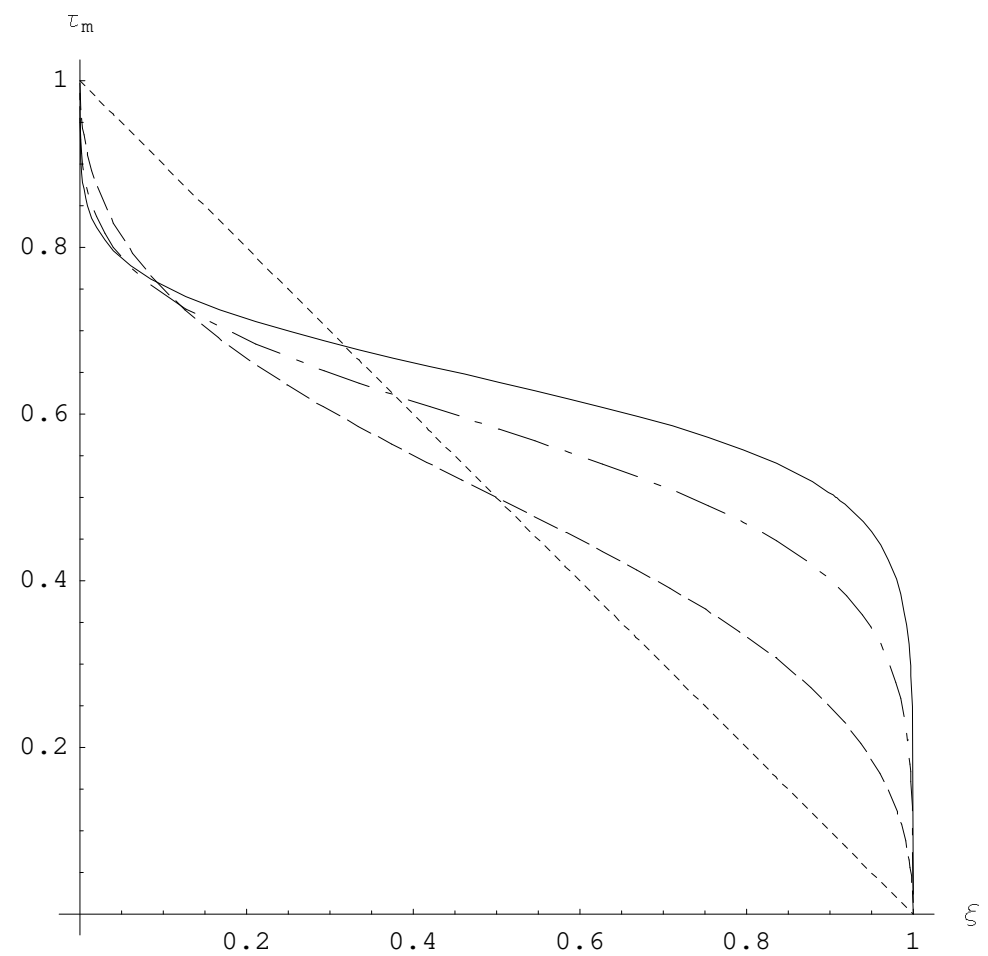

Fig. 1 